\title{
The Influence of Visual Models and Instructional Methods on the Development of Students' Graphic Representations
}

\author{
Jean Langan
}

\section{The Value of Drawing}

The value of drawing exists in art education as part of a unique symbolic domain that needs to be investigated in its own terms before one can establish similarities to other symbolic domains, for example to language, mathematics, or music (Golomb, 1992). According to Arnheim, the visual arts such as drawing, even in childhood, are grounded in a graphic representational logic and the language of drawing can and ought to be studied in its own right (p. 2).

Golomb points out that drawing is a uniquely human activity whose complex syntactic and semantic development can be studied systematically. Golomb sights the drawing performance as a truly creative activity of the child, who invents or reinvents in every generation, and across different cultures, a basic vocabulary of meaningful graphic representations. Thus, the way in which graphic representations are strung together to form compositions, how those graphic representations change for different age groups and through different methods of instruction and the significance of the drawing performance for students, is cardinal to art education.

Drawing is an activity that almost everybody does at one time or another (Wilson \& Wilson, 1982). The shared motives and reasons for drawing go from expressing an idea, to creating and inventing, to mastering a technique. The importance of drawing to the educational system, says Wilson, is three-fold. First, drawing is important because of its contribution to students' cognitive processes. Second, drawing contributes to students' competence and skill in the use of a valuable symbol system. Third, wellinstructed drawing will promote the acquisition of knowledge and understanding through a special visual mean pervaded with feeling and aesthetic quality. Therefore, drawing should be the principal studio activity.

Wilson indicates that many students equate drawing well with drawing accurately. Young people are influenced to be faithful to the model by the strong emphasis on visual realism in their graphic world. 
Accurate drawing, according to Wilson, is based on detailed information about the parts, structure, foreshortened appearance, etc.. How to incorporate this information to a two-dimensional surface must also be known to achieve accuracy in graphic representation.

\section{Basic Research on Drawing}

The basic research on drawing describes the conventional strategies of children's graphic representations. Cox (1986), reporting on the findings of Luquet $(1913,1927)$ notes that young children, ages' four to seven or eight years, are said to be in a stage of intellectual realism they draw what they know rather than what they see. In their drawing, they may include more of an object than they could possibly see from a particular viewpoint. When asked to draw a cup with its handle turned away and out of sight, five to seven year olds included a handle in their drawings of the cup (Freeman \& Janikoun, 1972).

Freeman (1980) suggests the possibility that child's drawing is knowledge-dominated only because the child lacks other specialized knowledge which would prevent that domination. In particular, the child may lack more complex or specialized skills. Freeman says knowing what something is does not guarantee that one know how it goes. In this case, the student may lack the more complex knowledge that allows the coordination of such skills as perspective and occlusion.

Piaget and Inhelder (1967) suggested that the child's early drawings are based on topical rather than projective relationships. Their representations of space do not exhibit Euclidean relations of proportion, length distance and shape and are unconcerned with the projective relations of perspective (Golomb 1992) It would follow that older students' drawings may then be based on complex projective alignments among objects. It is assumed that adults, in a stage of visual realism, will draw using a more comprehensive compositional strategy including the coordinated use of occlusion, proportion, viewing positions and linear perspective (Cox 1986).

However, studies such as Willats (1977a, 1977b), Duthie (1985) and Cox (1986) indicated that some individuals do not adopt a system of drawing in perspective even when they have reached the age when it is deveiopmentally possible. Linear perspective is rarely used before adolescence, and even then only infrequently (Golomb 1992). Other factors, then, such as less complex and less congruent drawing systems, must be considered in the graphic representations of studients. Three possible 
considerations would be; less advanced projection systems, occlusion, and temporal order of graphic representational strategies.

Willats (1977a, 1977b, 1985) conducted a series of studies that indicated one of the main types of drawing systems necessary to depict a hard-edged three-dimensional object is a projection system. In this system, the individual attempts to show how the object appears in space or how its angles project back into space. Willats presented the subjects with a scene consisting of a radio, a box, and a saucepan standing on a table. The table was arranged so that the subject faced one of the long sides. The radio was arranged so as partially to occlude the box, and all three objects occluded sections of the far edge of the table. The subject's point of view was controlled by the experimenter.

Willats found six classes of projection systems adopted by children of ages' five to seventeen to draw a three-dimensional object. Lines in the picture which represented edges in the scene normal to the picture were termed orthogonals. Willats defined class one, as no projection system; class two, as orthographic projection, in which the orthogonals cannot be depicted because the projections of edges normal to the picture plane appear as points; class three, as vertical oblique projection, in which the orthogonals appear vertical instead of horizontal; class four, as oblique projection, in which orthogonals remain parallel; class five, as naive perspective, in which the orthogonals converge between 20 and 60 degrees; and class six, as perspective, in which the orthogonal angle of convergence is 60 to 100 degrees. The drawing of the object will appear more realistic the higher it is on Willats's drawing scale.

In 1984, Chen, Therkelsen and Griffiths conducted a longitudinal study about the relationship between learning and representation drawing. They used the same six classes of projection systems as Willats (1977a, $1977 \mathrm{~b}$ ) for their criterion. Their findings about the representation of six to ten-year-old subjects supports the Willats studies.

Since we should not consider perspective drawing as a stagedependent natural endpoint of development (Golomb 1992), neither should we consider the acquisition of other complex drawing strategies to be stagedependent. Visual knowledge and techniques for graphic representation that lead to more sophisticated drawings must be disseminated through art instruction.

Kindler (1992) has pointed out the necessity of careful consideration of learning strategies which children spontaneously employ to solve 
problems of pictorial representation--Strategies such as occlusion and temporal order to name two. Projection systems have been looked at by Willats (1977a), Cox (1986) and Chen (1984), but there is a lack of information about other strategies.

The debate spans more than fitty years, regarding the innate logic of graphic representation imagery of children's drawings. It was at first shaped by the view that simplicity dictated the depth of the child's conception of the image. Now, there is growing evidence that children demonstrate a preference for complexity that goes beyond experience with a particular medium and that desire for complexity can convey (Freeman, 1980; Golomb, 1983a, 1983b)

The child's perception is more advanced than the child's drawing performance would indicate (Piaget, 1956; Golomb, 1992). There are difficulties for most children in the production of accurate graphic representations of objects (Willats, 1977a, 1977b, 1985; Freeman, 1980; Golomb, 1992).

\section{Implications for Art (Education) Instruction}

Wilson, Hurwitz, \& Wilson (1987) tell us that unless experience and education lead students, above fifth grade level, to transcend some of their biases, they will continue to use the same graphic solutions that young children, below fitth grade level, do, and may thus limit themselves to a lifetime of involuntary child-like graphic behavior.

The pedagogical implications of basic research in the drawing process, as discussed by Hagaman (1990), outline the theoretical framework for art education today. The suggestions seem to rest upon three premises:

1. Drawing should be the principal studio activity in school art programs.

2. Graphic models provide the most important contribution to the development of drawing ability.

3. The school drawing program should utilize exemplary works of art in providing such models.

The present study covers all three pedagogical suggestions. One, by using the drawing performance as a measure to gain information on instructional methods for art; two, by incorporating graphic models; and three, by utilizing exemplary works of art for drawing models. 
Studio performance makes up the largest proportion of instruction in the art classroom. This study will provide instructional knowledge in the area of drawing performance and production. Results of a two-dimensional model and a three-dimensional model on drawing instruction and student drawing performance will be examined. A reproduction of an exemplary work of art and a still life array of objects corresponding to the art work will be used as the two-dimensional and three-dimensional visual models.

Specifically, this study will examine the influence of direct modeling from visual models on the graphic representations of fifth grade, sixth grade and college level students. Conditions for the visual models will be two and three dimensional. The influence of the instructional training method of modeling drawing behavior on the graphic representations of this population will also be examined. Conditions for the instructional training method will be exposure or lack of exposure to the instructional training method. The independent variables of dimension and instructional methods will be manipulated by the experimenter.

Three age groups of students will form the population and complete a drawing task under varied conditions. The pilot test and group one participants will be college level students. Groups two and three will be sixth and fifth grade participants. The students being examined are untrained rather than highly trained in art. Their overall experience with art is limited, mostly to that which they have received in school. In the Urbana/Champaign area, the population would not necessarily be privileged to art instruction at the elementary level.

This study is concerned with the graphic representation strategies of fitth grade students and above, and the instructional methods that may positively influence those graphic representations. It expands on the research done by Willats (1985), adopting Willats' six-class projection system as a measuning instrument. Further, the study will record changes in the students graphic representations in the areas of occlusion and temporal order during the drawing performance. Information on appropriate instructional methods for fifth and sixth grade as well as college students will be reported

\section{Research Objectives.}

Research objective \#1 Providing visual models will lead the drawing performance of fifth and sixth grade and college level students toward more complex strategies of visual realism. 
Research objective \#2 The graphic representations of all groups will demonstrate the most complex strategies of visual realism in the condition of exposure to the two-dimensional visual model because the two-dimensional work has already solved the problems of translation from three dimensions to two dimensions.

Research objective \#3 The drawing performance of all groups will demonstrate more complex strategies of visual realism in the conditions of exposure to the instructional method of modeling drawing behavior.

Research objective \#4 Therefore, the drawing performance of groups one, two and three will show the most advanced strategies toward visual realism in the condition of exposure to the two-dimensional model with modeling drawing behavior.

\section{Method}

\section{Design}

A three by four, repeated measures research design will be used. There will be three groups of subjects in four conditions. The population will be drawn from University and elementary students. Group one will be composed of college aged students. Group two will be composed of sixth grade students and group three will be composed of fifth grade students. Condition one will be no modeling instruction with exposure to the twodimensional model. Condition two will be no modeling instruction with exposure to the three-dimensional model. Condition three will be modeling instruction with exposure to the two-dimensional model. Condition four will be modeling instruction with exposure to the three-dimensional model. Analysis of variance will be used to test for significance, correlation and interaction. The three main effects of age, modeling versus no modeling behavior and two-dimensional versus three dimensional models will be measured.

\section{Subjects}

The participants in this study will be students enrolled in Art Education 203, Art in the Elementary Grades, I, at the University of Illinois, students enrolled in the Saturday School art program at the University of Illinois and public school fifth and sixth grade students in the Urbana/Champaign, Illinois area. The subjects will be randomly selected from the available classes. The numbers of males and females will be approximately equal. 


\section{Materials}

Art Stimuli. Two-dimensional Model. The two-dimensional model used as the drawing stimulus in this study is an 11 " $\times 17^{\prime \prime}$ reproduction of the Master work of art, "Still Life with Letter to Thomas B. Clarke," by William M. Harnett, 1879. The total number of objects is the two-dimensional still life will be eight.

Art Stimuli. Three-dimensional Model. The three-dimensional model used as the drawing stimulus in this study will be a still-life array of objects corresponding in order and placement to the Master work of art, "Still Life with Letter to Thomas B. Clark," by William M. Harriet, (1879). The objects will display the same viewpoint, proportion, perspective and occlusion as did the two-dimensional model. The total number of objects in the three-dimensional still life will be eight.

Modeling Instruction. A video modeling the drawing behavior appropriate to this drawing task will be shown as an instructional method to each participant in condition three and condition four.

Drawing Task. Each participant will be given an 11 " $\times 17$ " sheet of white sulfite drawing paper and a soft lead pencil to use. Rulers and erasers will be available in the room.

\section{Procedure}

The first task of each participant will be to draw a still life array of objects from their imagination. The specific order and placement of the objects will be described in a set of printed instructions. These drawings will compile a baseline of information on the graphic representations of each participant.

Condition one of the experiment will follow the baseline task by about ten minutes. The following instructions will be given for conditions one and condition two: Draw the still life in front to you. Look at it very carefully. Draw it exactly as you see it in this model. Draw it the best way that you can. There is no time limit.

The same set of directions will be administered in conditions three and four with the addition of the video modeling drawing behavior.

\section{Analysis of Data}

The student drawings will be evaluated, categorized and rated under three major headings: drawing system, occlusion and temporal order. 


\section{References}

Chen, M. J., Therkelsen, M. \& Gritfiths, K. (1984). Representational Drawings of Solid Objects: A Longitudinal Study. Visual Arts Research, 1 o(19), 27-31.

Cox, M. (1986). Cubes are Difficult Things to Draw. British Journal of Developmental Psychology, 4, 341-345.

Duthie, R. (1985) The Adolescent's Point of View: Studies of Forms in Conflict. University Press.

Freeman, N. (1980) Strategies of Representation in Young Children. London: Academic Press.

Freeman, N. \& Janikoun, R. (1972). Intellectual Realism in Children's Drawings of a Familiar Object With Distinctive Features. Child Development, 4 3, 1116-1121.

Golomb, C. (1983a). Children's Graphic Planning Strategies and Early Principles of Spatial Organization in Drawing. Studies in Art Education, 24(2), 86-100.

Golomb, C. (1983b). On Imaginary of Real Decalages in Children's Representations: Compositional Trends in Drawing. Completion, and Selection Tasks. Visual Arts Research, 9(2), 71-81.

Golomb, C. (1992). The Child's Creation of a Pictorial World. Berkeley: University of California Press.

Hagaman, S. (1990). Recent Publications. Studies in Art Education, 31(3), 189-191.

Kindler, A. (1992). Worship of Creativity and Artistic Development of Young Children. University of British Columbia Press.

Luquet, G. H. (1913). Les Dessins d'un Enfant. Paris: Alcan.

Luquet, G. H. (1927). Le Dessin Enfantin. Paris: Alcan. 
Piaget, J. (1956).The Child's Conception of Space. London: Routledge and Kegan Paul.

Piaget, J. \& Inhelder, B. (1967). The Child's Conception of Space. New York: Norton.

Willats, J. (1977a). How Children Learn to Draw Realistic Pictures. Quarterly Journal of Experimental Psychology, 29, 367-382.

Willats, J. (1977b). How Children Learn to Represent ThreeDimensional Space in Drawings. In G. Butterworth (Ed.), The Child's Representation of the World (pp. 189-202). New York: Plenum Press.

Willats, J. (1985). Drawing Systems Revisited: The Role of Denotation Systems in Children's Figure Drawings. In Freeman, N. \& Cox, M. (Eds.), Visual Order (pp.78-100). Cambridge University Press.

Wilson, B. \& Wilson, M. (1982). Teaching Children to Draw: A Guide for Parents and Teachers. Englewood Cliffs, NJ: Prentice-Haii.

Wilson, B. \& Hurwitz, A., \& Wilson, M. (1987). Teaching Drawing From Art. Worcester, MA: Davis Publications. 